\title{
Time to increase WHO clinical stage of people living with HIV in public health facilities of Arba Minch town, south Ethiopia
}

\author{
Direslgne Misker Abyu ${ }^{1}$, Eskezyiaw Agidew Getahun ${ }^{1}$, Marelgn Tilahun Malaju ${ }^{1}$, \\ Habtamu Mellie Bizuayehu ${ }^{2}$ \\ ${ }^{1}$ Arba Minch University, Department of Public Health, Arba Minch, Ethiopia \\ ${ }^{2}$ Debre Markos University, Department of Public Health, Debre Markos, Ethiopia
}

\section{Email address:}

diresmisker@gmail.com (D. M. Abyu),marikum74@gmail.com (M. T. Malaju), habtamumellie@yahoo.com (H. M. Bizuayehu), esk1agid@gmail.com(E.A.Getahun)

\section{To cite this article:}

Direslgne Misker Abyu, Eskezyiaw Agidew Getahun, Marelgn Tilahun Malaju, Habtamu Mellie Bizuayehu. Time to Increase WHO Clinical Stage of People Living with HIV in Public Health Facilities of Arba Minch Town, South Ethiopia. Clinical Medicine Research. Vol. 3, No. 5, 2014, pp. 119-124. doi: 10.11648/j.cmr.20140305.11

\begin{abstract}
Background: Highly Active Antiretroviral Therapy improves time to death and recurrence of any opportunistic infections in peoples living with HIV. But little is known about its effect on time to increase WHO clinical stage. Thus, this study was aimed at assessing time to increase WHO clinical stage and associated factors. Method: Retrospective cohort study was used and the required sample size was 464. Study participants were selected randomly from the list of adult people living with HIV attending the public health facilities for ART. Univariate analysis was used to describe patients' baseline and follow up characteristics. Kaplan-Meier survival and log rank test were used to estimate survival and compare survival curves respectively. Cox proportional-hazard regression model was used to calculate hazard rate and to determine independent predictors of time to increase WHO clinical stage. Result: A total of 464 patients (232 in each cohort) contribute for 898.12 person years of follow up. The overall incidence rate of increased WHO clinical stage was 16.6 per 100 person years of follow up and the incidence of increased WHO clinical stage was 38.5 per 100 person year and 3.8 per 100 person year of follow up in ART and HAART cohorts respectively. Being on Highly Active Antiretroviral Therapy and taking prophylaxis at baseline were the identified predictors' that prevent increment of WHO clinical stage. In contrary being widowed and single marital status, substance use, rural residence and being in age category 45 years and above were independent predictors for increment of WHO clinical stage. Conclusion: Increment of WHO clinical stage was higher in pre ART cohorts. Special consideration should be given for those who are widowed, substance users and aged 45 years and above.
\end{abstract}

Keywords: PLWHIV, WHO Clinical Stage, Increase, Public Health Facility

\section{Introduction}

According to the latest United Nation's Agency for International Development (UNAID) estimate, about 34 million people were living with human immunodeficiency virus (HIV) in 2011 [1,2]. Globally, the annual number of people dying from acquired immunodeficiency syndrome (AIDS)-related causes was 1.7 million in 2011 [3].

According to 2011 Ethiopian demographic health survey, the overall national adult (age 15-49 years) HIV prevalence was $1.5 \%$ (1\% among males and $1.9 \%$ among females) and
$0.9 \%$ for SNNPR [4]. Introduction of combination antiretroviral therapy in 1996 has led to dramatic reductions in morbidity and mortality from HIV/AIDS [1, 2]. In Ethiopia, the fee based and universal free access antiretroviral (ARV) treatment was started in 2002/3 and $2004 / 5$ respectively $[5,6]$.

The WHO clinical staging system for HIV/AIDS was developed in 1990 and revised in 2007. It uses clinical parameters to classify subjects into any one of four categories i.e. stage 1 to IV, progressing from primary HIV infection to advanced HIV/AIDS. It is these categories that are used to guide decision making for the management of 
HIV/AIDS patients where there is limited access to laboratory services [7]. These clinical guidelines are readily available, convenient to the patient, cheap and can be applied by trained clinicians even in the most remote health facilities

\section{Methods and Materials}

\subsection{Study Area and Study Design}

Arba Minch town has one General hospital and one public health center, which provide ART service. There were 4759 adult PLWHIV in Arba Minch Hospital and Health center on chronic HIV care of these 2143 were between November 27, 2009 and January 24, 2014. Retrospective cohort study design was used to assess time to increase WHO clinical stage of PLWHIV.

\subsection{Source and Study Populations}

All 15 years old and above PLWHIV having chronic HIV care or on ART follow up in Public Health facilities of Arba Minch town providing ART service were the source populations. All 15 years old and above PLWHIV having chronic HIV care or on ART follow up in Public Health facilities of Arba Minch town providing ART service from November 27, 2009 to January 24, 2014 were study populations.

\subsection{Sample Size Determination and Sampling Procedure}

The sample size was calculated based on the assumption of $95 \%$ confidence interval and $80 \%$ power for two population proportion formula with one to one ratio for HAART and pre- ART PLWHIV using the proportions of OIs, $74.2 \%$ for Pre ART and $25.8 \%$ HAART PLHIV respectively [10]. Epi info version 3.5.1 is used to calculate the sample size.

$$
\begin{gathered}
n_{1}=\frac{\left[Z_{\frac{\sigma}{2}} \sqrt{\left(1+\frac{1}{r}\right) P(1-P)}+Z_{\beta} \sqrt{P_{1}\left(1-P_{1}\right)+\frac{P_{2}\left(1-P_{2}\right)}{r}}\right]^{2}}{\left(P_{2}-P_{2}\right)^{2}} \\
\mathrm{P}=\frac{P_{1}+r P_{2}}{1+r} \quad r=\frac{n_{2}}{n_{1}}
\end{gathered}
$$

Where:

$\mathrm{n} 1=$ number of HIV infected patients who are Pre ART (non exposed)

$\mathrm{n} 2=$ number of HIV infected patients who are on ART (Exposed)

$\mathrm{r}=$ the ratio of exposed to non exposed HIV infected patients $=1$

$\mathrm{P} 1=$ proportion of OIs in HAART receiving HIV infected patients

$\mathrm{P} 2=$ proportion of OIs in Pre ART HIV infected patients

$\mathrm{Z} \alpha / 2: 1.96$ at $95 \%$ confidence interval

Accordingly, the calculated sample size was 422 (211 for HAART and Pre ART); after adding $10 \%$ contingency the total sample size was 464,232 patients for each cohort

\subsection{Sampling Procedures}

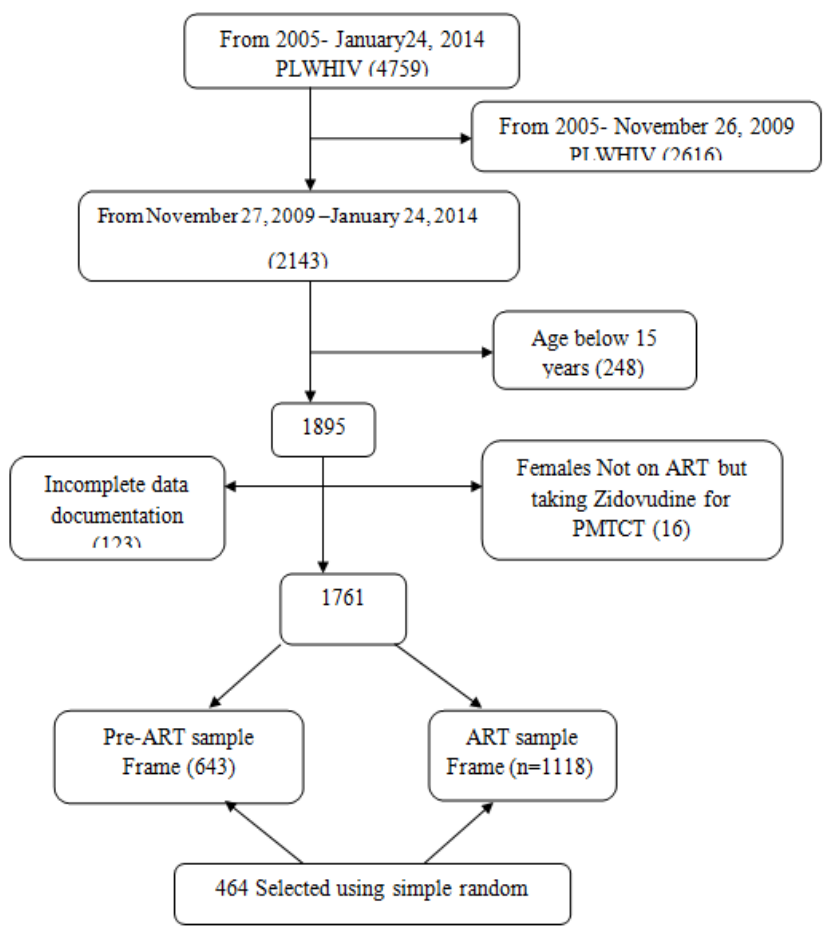

Figure 1. Schematic presentation of sampling procedure of PLWHIV enrolled for cohort between $27^{\text {th }}$ November, 2009 and $24^{\text {th }}$ January, 2014 in public Health facilities of Arba Minch town, Southern Ethiopia, 2014

\subsection{Data Collection Tools, Procedures and Quality Control}

Data collection format was developed from federal ministry of health HIV care/ART follow up form which is used in the ART clinic and also the patients' card; the format includes the following check list:

- socio-demographic characteristics (sex, age, residence, marital status, occupational status, educational status);

- $\quad$ ART and chemoprophylaxis information (ARV drug treatment, drug adherence, line of treatment regimen, prophylaxis treatment and its adherence);

- laboratory information (CD4 count, hemoglobin value);

- Clinical information (WHO clinical staging, weight, height); and

- Functional status of patients (working, ambulatory, bedridden).

The data was collected by reviewing HIV care/ART follow up form, laboratory requests and patients' card. Clients with WHO clinical stage increased was confirmed by reviewing patients HIV care/ART follow-up form or patient card. Individuals who change from unexposed to exposed, drop-out/loss to follow-up/transferred out/dead by any disease without increased WHO clinical stage at the end of the study period were censored. The outcome of each subject was dichotomized into censored or increase WHO clinical stage. 


\subsection{Data Quality Management, Processing and Analysis}

To ensure the quality of data, data collectors were trained nurses, recruited from ART clinic on method of extracting the needed data from patient's records and filling on data collection format. On the days of data collection, the principal investigator and the supervisor supervises the data collection process by checking completeness of the data. Three data collectors and one supervisor who had direct experience and working on ART clinic was recruited for data collection and supervision respectively. Data collectors and supervisor was trained on objectives of the study, selection of exposed and unexposed, how to keep confidentiality of information, the contents of the questionnaire and data quality management by the principal investigator. The data abstraction form was checked for completeness and consistency by the principal investigator before data entry. Completed data abstraction format was coded by numbers and entered in computer software EPI info version 3.5.1 statistical package and exported to SPSS version 16 and STATA version 11 for analysis. Crosschecking and data cleaning was done for accuracy and consistencies carried out by running frequencies of each variable by principal investigator. The risk of developing OIs among patients with retrospective follow up in Pre ART or HAART cohort was assessed using the person-year method. Incidence rate was calculated as number of cases per 100 person years observed. Before running the Cox regression model assumption of proportional-hazard was checked by Schoenfeld residual and log minus log plots. Cox proportional hazards model was used to assess predictors of increase WHO clinical stage, the KaplanMeier technique and the generalized log-rank test were used to construct and compare the WHO clinical stage increment free survival probabilities curves of the two groups.

\subsection{Study Variables}

\subsubsection{Dependent Variable}

- WHO clinical stage increment

\subsubsection{Independent Variable}

- Exposure variable: HAART status (HAART initiation or not)

- Socio demographic (Age, sex, education status, marital status, occupation status)

- Clinical and other variables ( weight, CD4+ count, prophylaxis, Hemoglobin level, adherence level for HAART)

\subsection{Operational Definition}

Increase WHO clinical stage: PLWHIV who had clinical stage higher than the baseline clinical stage

Pre ART PLHIV: The person whose HIV positive status confirmed but not eligible for ART;

HAART PLWHIV: The person whose HIV positive status confirmed and started ART;

Survival: The person without increased WHO clinical stage

Censored: No WHO clinical stage increment in study participant during follow-up study but not sure for future increment;

Drop out: If a PLWHIV on HIV care lost to follow up for more than three months as recorded by ART clinic personnel;

Lost to follow up: If PLWHIV on HIV care not seen for equal to or more than one month as recorded by ART clinic personnel;

Transferred out: If PLWHIV on HIV care in one health institution shift to other health institution;

\subsection{Ethical Considerations}

Ethical clearance was obtained from the Ethical Review Committee of ACIPH before conducting the study. Arba Minch Hospital and Health center was informed about the objective of the study through a support letter from Arba Minch University, department of Public Health and permission was obtained from the Hospital and Health center administrations before starting data collection. To keep the confidentiality personal identifiers were not included in the data abstraction format.

\section{Result}

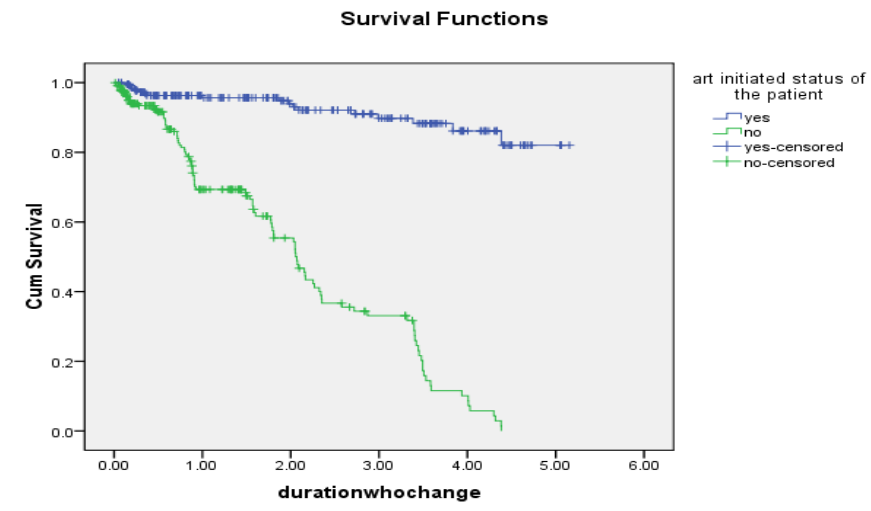

Figure 2. Kaplan-Meier estimate of WHO clinical stage increment free survival with and without ART, in public Health facilities of Arba Minch town, Ethiopia, 2014 
In this retrospective cohort study there were 464 participants each consisting 232 were followed for a total of 757.53 person years and median of 1.23 person year. The WHO clinical stage increment free survival person year follow up was 476.82 and 280.71 in HAART and Pre ART cohorts respectively.

\subsection{Socio-Demographic Characteristics}

In this study, 297 (64\%) females and 167 (36\%) males were participated. More than one third $(38.1 \%)$ of the participants were in the age range between 25 and 34 years with median age of $30(\mathrm{IQR}=24-37)$. Most of the study participants were urban by residence $365(78.7)$, married 245(52.8) and employed 293(63.1).

\subsection{Clinical and Laboratory Characteristics}

At base line, most of the study participants 436 (94\%) and $416(89.7 \%)$ had working functional status at base line and follow up respectively. Two hundred seventy nine $(60.1 \%)$ of the participants were taken prophylaxis at baseline and around $28 \%$ of the participants had CD4 count between 200-350 cells/micro liter both at baseline and follow up. Half of the study participants were taking HAART at baseline and follow up. During follow up $126(27.2 \%)$ had increased WHO clinical stage, 31(6.7\%) lost to follow up/drop out, 22(4.7\%) transfers out, 9(1.9) deaths and 276(59.5\%) HIV positive people were censored.

Table 1. The actuarial life table estimation of cumulative incidence of increased WHO clinical stage in PLWHIV in public Health facilities of Arba Minch town, between November 27, 2009 and January 24, 2014

\begin{tabular}{|c|c|c|c|c|c|c|}
\hline $\begin{array}{l}\text { ART } \\
\text { initiated }\end{array}$ & $\begin{array}{l}\text { Interval Start } \\
\text { Time }\end{array}$ & $\begin{array}{l}\text { Number Entering } \\
\text { Interval }\end{array}$ & $\begin{array}{l}\text { Number } \\
\text { Withdrawing during } \\
\text { Interval }\end{array}$ & $\begin{array}{l}\text { Number of Terminal } \\
\text { Events }\end{array}$ & $\begin{array}{l}\text { Cumulative } \\
\text { Proportion Surviving } \\
\text { at End of Interval } \\
\end{array}$ & Hazard Rate \\
\hline \multirow{5}{*}{ Yes } & {$[0-1)$} & 232 & 75 & 9 & .95 & .05 \\
\hline & {$[1-2)$} & 148 & 41 & 2 & .94 & .02 \\
\hline & {$[2-3)$} & 105 & 30 & 4 & .90 & .05 \\
\hline & {$[3-4)$} & 71 & 36 & 2 & .86 & .04 \\
\hline & {$[4-5)$} & 33 & 27 & 1 & .82 & .05 \\
\hline \multirow{5}{*}{ No } & {$[0-1)$} & 232 & 83 & 51 & .73 & .31 \\
\hline & {$[1-2)$} & 98 & 33 & 14 & .61 & .19 \\
\hline & {$[2-3)$} & 51 & 5 & 20 & .36 & .52 \\
\hline & {$[3-4)$} & 26 & 3 & 16 & .12 & .97 \\
\hline & {$[4-5)$} & 7 & 0 & 7 & .00 & 0.99 \\
\hline
\end{tabular}

Table 2. Bivariate and multivariate Cox proportional hazard analysis for Predictors of increased WHO clinical stage among HIV infected patients in public Health facilities of Arba Minch town, 2014

\begin{tabular}{|c|c|c|c|c|c|}
\hline Covariates & No at risk & NO increased WHO clinical stage & CHR(95\% CI) & AHR( 95\% CI) & P value \\
\hline \multicolumn{6}{|c|}{ HAART Status } \\
\hline HAART & 232 & 18 & $0.07(0.04,0.13)$ & $0.03(0.01,0.06)$ & $<0.001^{*}$ \\
\hline Pre-ART & 232 & 108 & 1 & 1 & \\
\hline \multicolumn{6}{|l|}{ Sex } \\
\hline Male & 167 & 71 & $2.57(1.80,3.66)$ & $1.30(0.84,2.01)$ & 0.24 \\
\hline Female & 297 & 55 & 1 & 1 & \\
\hline \multicolumn{6}{|c|}{ Marital status } \\
\hline Married & 245 & 63 & 1 & 1 & \\
\hline Single & 58 & 28 & $2.68(1.69,4.25)$ & $7.42(3.50,15.70)$ & $0.001 *$ \\
\hline Divorced & 81 & 10 & $0.84(0.43,1.63)$ & $1.98(0.94,4.19)$ & 0.073 \\
\hline Widowed & 80 & 25 & $1.42(0.89,2.27)$ & $9.03(4.84,16.83)$ & $0.001 *$ \\
\hline \multicolumn{6}{|c|}{ Age category } \\
\hline $15-24$ & 121 & 29 & 1 & 1 & \\
\hline $25-34$ & 177 & 56 & $0.60(0.38,0.95)$ & $1.96(0.96,4.01)$ & 0.067 \\
\hline $35-44$ & 121 & 34 & $0.48(0.29,0.81)$ & $1.37(0.61,3.08)$ & 0.453 \\
\hline$>=45$ & 45 & 7 & $0.29(0.13,0.66)$ & $3.05(1.02,9.10)$ & $0.045^{*}$ \\
\hline \multicolumn{6}{|c|}{ Supportive care } \\
\hline Present & 166 & 63 & $0.30(0.21,0.44)$ & $0.88(0.57,1.37)$ & 0.573 \\
\hline Absent & 298 & 63 & 1 & 1 & \\
\hline \multicolumn{6}{|c|}{ Prophylaxis given } \\
\hline Yes & 279 & 55 & $0.37(0.26, .53)$ & $0.39(0.23,0.65)$ & $<0.001 *$ \\
\hline No & 185 & 71 & 1 & & \\
\hline \multicolumn{6}{|l|}{ Address } \\
\hline Urban & 365 & 77 & 1 & 1 & \\
\hline Rural & 99 & 49 & $2.28(1.58,3.28)$ & $1.87(1.08,3.23)$ & $0 . .025^{*}$ \\
\hline \multicolumn{6}{|c|}{ Substance use } \\
\hline Yes & 82 & 35 & $3.58(2.39,5.34)$ & $3.99(2.37,6.73)$ & $<0.001 *$ \\
\hline No & 382 & 91 & 1 & 1 & \\
\hline
\end{tabular}

$*=$ statistically significant 
The Kaplan Meier curve showed that taking HAART increased WHO clinical stage increment free survival with log rank P-value $<0.001$ than the pre ART cohort.

\subsection{Predictors of OIs Incidence}

To identify independent predictors of increased WHO clinical stage, a multivariate Cox-Proportional hazard adjusted model was fitted with the variables having a $\mathrm{P}$ value less than 0.2 in the bivariate analysis. (See table 2)

\section{Discussion}

In this retrospective cohort study, more than one fourth of the participants had increased WHO clinical stage. The proportion of increased WHO clinical stage was significantly different $(\mathrm{p}<0.001)$ between Pre-ART and HAART PLWHIV.

The overall incidence of increased WHO clinical stage was 16.6 per $100 \mathrm{PY}$. It was higher compared to the results from South Africa 8.52/100 PY and lower from $\mathrm{Co}^{\wedge}$ te d'Ivoire $36.8 / \mathrm{PY}$. These could be explained as a result of the study in South Africa considers only severe HIV related illness so which might have underestimation of the overall morbidity density rate or increased WHO clinical stage. The result from $\mathrm{Co}^{\wedge}$ te d'Ivoire shows higher rate of opportunistic infections because of the study includes a wide range of bacterial disease which were not included in this study so which might have overestimated the incidence density rate $(22,23)$.

The incidence of increased WHO clinical stage showed a decline from 38.5/100 PY (95\%CI: 31.7- 46.3) in those Pre ART patients to $3.8 / 100 \mathrm{PY}(95 \% \mathrm{CI}: 2.31-5.85)$ in those using HAART. Which is lower than a study conducted in Brazil 113.73/100 PY (95\%CI: 102.24-126.46) in those patients not using HAART to $41.30 / 100$ using HAART. This is due to the study conducted in Brazil includes children which may have lower immunity and leads to increased WHO clinical stage and but the current study includes only adults.

In the present study, the introduction of HAART is associated with a dramatic effect in the overall reduction of incidence rates of increased WHO clinical stage in PLWHIV which is supported by different studies [10, 14, 15, 16 and 17]. A study in Côte d'Ivoire shows Patients who had been on HAART for $>6$ months were 91\% (RR 0.09 , 95\% CI 0.04-0.19) less likely to die than patients followed on cotrimoxazole alone (14). Taking Prophylaxis decreased increment of WHO clinical stage by $61 \%$. This is in line with a study in South Africa (19).

In analysis of predictor variable patient with widowed and single marital status was nearly two times at higher risk of developing increased WHO clinical stage than patients with marred marital status. This is possibly because of having supporter for timely taking of medication as directed. Male gender was associated with higher incidence of increased WHO clinical stage with $\mathrm{p}<0.001$ which is in congruent with a study in resource limited settings.

Being substance user was nearly four times higher risk than non users. This may be due to substance use may cause to take subscribed medications untimely. Rural residence increased occurrence of increased WHO clinical stage nearly two times than being urban residence. This may be due to the fact that urban residence enables for easy access of information how to prevent occurrence of diseases and treatment purpose.

\section{Limitations and Strengths of the Study}

Strength- Since we used trained data collectors these decreased the occurrence of misclassification bias

Limitation- Since the study is based on secondary data, some predictor variables are not completely registered and not consistent in chronic care follow up chart and medical registration. Misclassification bias may also be introduced

\section{Conclusions}

The overall incidence rate of increased WHO clinical stage was 16.6 per $100 \mathrm{PY}$. The incidence of increased WHO clinical stage showed a decline from 38.5/100 PY in those patients not using HAART to $3.8 / 100 \mathrm{PY}$ in those using HAART. Independent significant predictors that increase occurrence (risk factors) and improve survival (preventive factors) were identified. Those risk factors were being widowed and single than married, substance use, rural residence and age 45 years and above; however, the preventive factors were taking HAART and prophylaxis.

\section{Recommendations}

\section{To Hospitals and Health Centers with ART Clinic (Giving HIV Care and Support)}

- Special consideration should focus on high risk groups such as widowed and substance users and in the elderly especially in those 45 years and above.

\section{To Research and Academic Institutions}

- Further observational studies with prospective design to ascertain the findings are recommended.

\section{Abbreviations}

ART-Anti Retroviral Therapy, HAART-Highly Active Anti Retroviral Therapy, WHO-World Health Organization

\section{Acknowledgment}

We would like to acknowledge Arba-Minch University for granting the fund to undertake this study. We are also 
thankful to the heads of health facilities where the study is conducted.

\section{Authors' Contributions}

DMA was investigator, involved in proposal writing, designing, and recruitment and training of supervisors and data collectors, analysis and write-up and in all stages of the project implementation. MTM contributed in the designing of the methodology, write-up and involved in the final approval of the paper. HMB contributed in the designing of the methodology, write-up and involved in the final approval of the paper. All authors read and approved the final manuscript. EAG contributed in the designing of the methodology, write-up and involved in the final approval of the paper. All authors read and approved the final manuscript.

\section{References}

[1] U.S. Global Health Policy Fact Sheet the Global HIV/AIDS Epidemic, 2011.

[2] WHO Global HIV/AIDS Response: Epidemic update and health sector progress towards Universal Access WHO, UNICEF UNAIDS, 2011.

[3] UNAIDS Together we will end AIDS. Joint United Nations Programme on HIV/AIDS, Geneva, 2012.

[4] Central Statistical Agency (CSA), Ethiopia Demographic and Health Survey; final draft report. Addis Ababa Ethiopia, ICF International Calverton, Maryland, USA. 2011.

[5] Federal HIV/AIDS Prevention and Control Office Annual Performance Report of Multi-sectoral HIV/ AIDS Response, Federal HIV/AIDS Prevention and Control Office, 2010.

[6] Report on progress towards implementation of the UN Declaration of Commitment on HIV/AIDS. Federal Democratic Republic of Ethiopia Federal HIV/AIDS Prevention and Control Office.

[7] WHO, Interim WHO Clinical Staging of HIV/AIDS and HIV/AIDS Case Definitions for surveillance in Africa Region, 2005.02.

[8] Mariam ZT, Abebe G, Mulu A. Opportunistic and other intestinal parasitic infections in AIDS patients, HIV seropositive healthy carriers and HIV seronegative individuals in southwest Ethiopia. East Afr J Public Health, 2008, 5: 169-173.

[9] Gallant JE, Moore RD, Chaisson RE. Prophylaxis for opportunistic infections in patients with HIV infection. Ann Intern Med, 1994, 120: 932-944.

[10] Candiani TM, Pinto J, Cardoso CA, Carvalho IR, Dias AC. et al. Impact of highly active antiretroviral therapy (HAART) on the incidence of opportunistic infections, hospitalizations and mortality among children and adolescents living with HIV/AIDS in Belo Horizonte, Minas Gerais State, Brazil. Cad Saude Publica. 2007;23
[11] Missaye A, Dagnew M, Alemu A, Alemu A. Prevalence of intestinal parasites and associated risk factors among HIV/AIDS patients with pre-ART and on-ART attending dessie hospital ART clinic, Northeast Ethiopia. AIDS Res Ther. 2013 Feb 25;10(1)

[12] Sun HY, Chen MY, Hsieh SM, Sheng WH, Chang SY. et al. Changes in clinical spectrum of opportunistic illnesses in persons with HIV infection in Taiwan in the era of HAART.. J. Infect. Dis, 2006; 59, 311-316.

[13] Manosuthi W, Chaovavanich A, Tansuphaswadikul S, Prasithsirikul W, Inthong Y. et al. Incidence and risk factors of major opportunistic infections after initiation of antiretroviral therapy among advanced HIV-infected patients in a resource-limited setting. J Infect. 2007 Nov;55(5)

[14] Losina E, Yazdanpanah Y, Wang B, Messou E, Anglaret X. et al. The independent effect of highly active antiretroviral therapy on severe opportunistic disease incidence and mortality in HIV-infected adults in Côte d'Ivoire. Antivir Ther.2007; 12(4): 543-551.

[15] Jerene D, Naess A, Lindtjorn B. Antiretroviral therapy at a district hospital in Ethiopia prevents death and tuberculosis in a cohort of HIV patients. AIDS, 2006; 3:10.

[16] Ghate M, Deshpande S, Tripathy S, Nene M, Gedam P. et al. Incidence of common opportunistic infections in HIVinfected individuals in Pune, India: analysis by stages of immune suppression represented by CD4 counts. International Journal of Infectious Diseases. 2009;13

[17] Badri M WD, Wood R. Effect of highly active antiretroviral therapy on incidence of tuberculosis in South Africa: a cohort study. Lancet 2002;359: 2059-64

[18] Kassa D. The pattern of immunologic and virologic responses to Highly Active Antiretroviral Treatment (HAART): Does success bring further challenges? Ethiop. J. Health Dev. 2011;25(1):61-70

[19] Charles B. Wood R, Badri M, Zilber S, Wang B. et al. CD4 Decline and Incidence of Opportunistic Infections in Cape Town, South Africa: Implications for Prophylaxis and Treatment. J Acquired Immune Deficiency Syndrome. 2006,42 (4)

[20] Kibret KT, Yalew AW, Belaineh BG, Asres MM. Determinant Factors Associated with Occurrence of Tuberculosis among Adult People Living with HIV after Antiretroviral Treatment Initiation in Addis Ababa, Ethiopia: A Case Control Study. 2013. doi:10.1371

[21] Biadgilign S, Reda A and Digaffe T. Predictors of mortality among HIV infected patients taking antiretroviral treatment in Ethiopia: a retrospective cohort study. AIDS Research and Therapy, 2012, 9:15

[22] Badri M, Ehrlicha R, Wood R, et al. Initiating cotrimoxazole prophylaxis in HIV-infected patients in Africa: an evaluation of the provisional WHO/UNAIDS recommendations. AIDS. 2001,15:1143-1148.

[23] Anglaret X, Messou E, Ouassa T, et al. Pattern of bacterial diseases in a cohort of HIV-1 infected adults receiving cotrimoxazole prophylaxis in Abidjan, $\mathrm{Co}^{\wedge}$ te d'Ivoire. AIDS2003, 17:575-584. 